\title{
Stability of Surface State Electrons on Helium Films
}

\author{
P. Leiderer ${ }^{1}$ - E. Scheer ${ }^{1}$ - K. Kono ${ }^{2,3}$. \\ J.-J. Lin' ${ }^{2,3,4}$. D. G. Rees ${ }^{2,3}$
}

\begin{abstract}
Electrons on helium substrates form a model Coulomb system in which the transition from classical electron liquid to Wigner crystal is readily observed. However, attempts to increase the electron density in order to observe the 'quantum melting' of the system to a Fermi degenerate gas are hindered by an instability of the helium surface. Here we describe experimental efforts to reach the degenerate regime on thin helium films and microstructured substrates, for which the surface instability is suppressed. We demonstrate that, although the electron densities obtained exceed those for bulk helium substrates, observation of quantum melting remains challenging. We discuss possible solutions to the technical challenges involved.
\end{abstract}

Keywords Electrons on helium · Thin films · Quantum melting

\section{Introduction}

Surface state electrons (SSE) on liquid helium form a unique 2-dimensional (2D) Coulomb system under particularly clean and well-defined conditions, and their interesting properties have been described in several reviews [1] (here we discuss ${ }^{4} \mathrm{He}$ exclusively). The SSE phase diagram, as calculated by Peeters and Platzman [2],

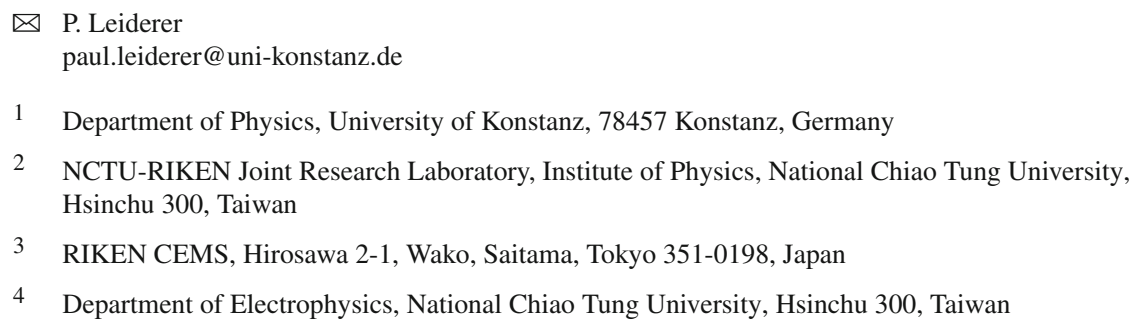


is dominated by a competition between three energy contributions: (i) the thermal energy, proportional to temperature, $E_{\text {th }} \sim T$; (ii) the Coulomb energy $E_{\text {Coul }}$ resulting from the mutual repulsion of the charges, which is proportional to the inverse distance between the electrons and hence $\sim n^{1 / 2}$ (where $n$ is the electron density); (iii) the Fermi energy, which for a $2 \mathrm{D}$ system is $E_{\mathrm{F}} \sim n$. Accordingly, the phase diagram of such a system displays three regimes, namely a classical 2D Coulomb gas/fluid, a Wigner crystal phase, and a degenerate Fermi gas, which is predicted to appear for $n>2.4 \times 10^{12} \mathrm{~cm}^{-2}[2]$.

As yet, the quantum melting transition from the Wigner crystal to the degenerate Fermi remains essentially unexplored due to an electro-hydrodynamic (ehd) instability of the bulk liquid surface at an electron density of $2 \times 10^{9} \mathrm{~cm}^{-2}$ [3]. Above this density (which is reached at an applied electric field $E_{\text {crit }} \approx 3 \mathrm{kV} \mathrm{cm}^{-1}$ ) the homogeneous density distribution of the SSE breaks up into a lattice of multielectron dimples. On further increase of the applied electric field, the electrons in the dimples break through the liquid surface as multielectron bubbles [4-7]. This ehd instability of the SSE can be suppressed if, instead of liquid helium, a solid with negative electron affinity such as hydrogen or neon is used as a substrate [8]. In the present paper, we discuss another approach to suppress the ehd instability and to reach higher electron densities, namely the use of thin helium films instead of bulk liquid helium as a SSE substrate.

\section{Stability of Electrons on Helium Films}

Helium films are more stable against deformations of their surface than the bulk liquid because of the van der Waals forces of the substrate. Saturated films in equilibrium with the bulk liquid have a thickness $d$ which depends on the height $h$ of the film above the liquid level as $d \sim h^{-1 / 3}$ (in the non-retarded regime). When such a film is charged with electrons, its thickness is reduced due to the electrostatic pressure of the SSE sheet, an effect which may be used to determine the electron density on the helium film [9]. Since the solid substrate underneath the helium film contributes to the image charge of the SSE and hence to a partial screening of the Coulomb interaction, the calculated area of the Wigner crystal regime on films in the $(n-T)$ phase diagram shrinks [2]. Experiments with SSE on films have been carried out both on insulating and on metallic substrates:

\subsection{SSE on Insulating Substrates}

The first experiments showing an enhanced stability of He films compared to the bulk liquid were performed by Etz et al. [9], and the highest density reached on various insulating materials was $10^{11} \mathrm{~cm}^{-2}$, i.e., close to the regime where quantum melting on films is expected. However, in these measurements, the state of the SSE was not determined. Jiang et al. [10] then investigated the classical region of the phase diagram of the Wigner crystal on He films up to a density of $2 \times 10^{10} \mathrm{~cm}^{-2}$, and found good agreement with the predictions for screened Coulomb interaction. This was confirmed later by Mistura et al. [11]. 
Results for higher electron densities, up to $10^{11} \mathrm{~cm}^{-2}$, were obtained by Günzler et al. [12] on a $\mathrm{He}$ film supported by a $500 \mathrm{~nm} \mathrm{SiO} 2 / \mathrm{Si}$ substrate. In this experiment, the microwave absorption by a sheet of SSE in a cavity was determined at a frequency of $10 \mathrm{GHz}$ as a function of the electron density. After an initial increase of the absorption proportional to the electron density, a kink was observed that signaled the transition to the Wigner crystal phase. Upon a further increase of $n$ the absorption became nearly constant, and then increased again steeply. This second feature was interpreted as a signature of quantum melting, but detailed investigations in this density range, which would allow a more conclusive statement, have proven challenging. At these high electron densities, the helium film is already very thin, and the electrons easily are lost through the film, forming a space charge on the substrate that hampers the experiments.

In our search for suitable substrates for attaining high electron densities, we have developed a simple, quick method in order to characterize samples: First electrons are supplied in a regular pulse train from a heated filament. As the helium film charges up, the amplitude of the current pulses decreases exponentially, because the SSE sheet screens the electric field between film and electron source more and more. After the film is charged to saturation, the SSE are removed sideways by a high voltage applied to a pick-up wire. As long as all the deposited electrons remain on top of the He film and are mobile, this charging/discharging sequence can be reproducibly repeated. If on the other hand charges become localized, and in particular when they traverse the He film, either due to an instability or by tunneling, higher and higher voltages have to be applied to the bottom electrode underneath the insulator substrate to observe charging pulses, as in Fig. 1. With this method, we are presently studying various dielectric and semiconducting materials in order to check their suitability as substrates for high electron densities. This includes also $\mathrm{Si}$ wafers with a thin $(1 \mathrm{~nm})$ natural oxide, for which one might hope that the charging effects described above are reduced. However, preliminary results indicate that this unfortunately does not seem to be the case.

\subsection{SSE on Metallic Substrates}

On metallic substrates, the charging up by electrons which traverse the film can be avoided. We have therefore also studied SSE on thin He films supported by $45 \mathrm{~nm}$-thick

Fig. 1 Charge registered by the bottom electrode underneath the insulating substrate as a function of time. At the beginning electrons from the previous charging process are discharged. Then the film is charged again in a series of pulses (Color figure online)

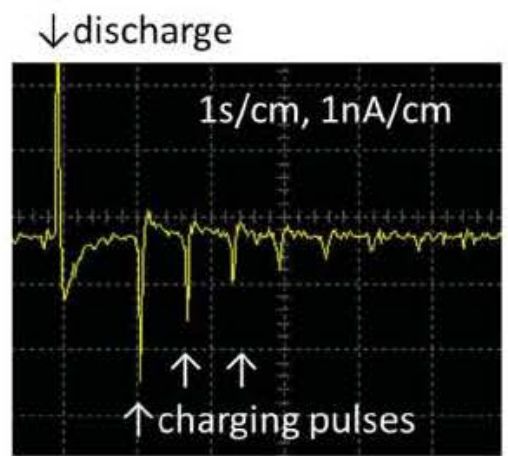


evaporated gold layers on glass [13]. No adhesion layer was deposited between the gold and the glass. AFM measurements revealed a 'hilly' surface profile with a typical amplitude of several $\mathrm{nm}$ and a lateral spacing of order $100 \mathrm{~nm}$. The SSE density was determined via a measurement of the film thickness by surface plasmon resonance. In Ref. [13] (Fig. 3) the film was initially charged to an electron density of $2 \times 10^{10} \mathrm{~cm}^{-2}$, and then the electron source was switched off. In contrast to insulating substrates, the electron density began to drop immediately, first exponential-like, and then linearly over a long timescale. This basic behavior was found in all the samples measured. The reason for the linear behavior is not yet understood, but it might provide a clue for developing models for the electron loss through the He film. One might expect that the sample roughness plays the dominant role. However, although the quality of the sample is of influence, the history of the charging is also important.

We demonstrated this by using two gold films, denoted as the upper and lower segments, that were arranged in parallel and separated by a narrow gap of $100 \mu \mathrm{m}$ [14]. The He film above the upper segment was charged in the usual way by electron pulses from a hot filament, while the lower segment was kept at a negative voltage. The electron densities on both segments were monitored simultaneously via the He film thickness, as before. Immediately after charging the upper segment to a density of $10^{11} \mathrm{~cm}^{-2}$, a potential difference of $120 \mathrm{~V}$ was applied between the two segments, in order to pull electrons from the upper segment to the lower segment. The experimental traces for the two segments are shown in Fig. 2. The density on the lower segment, which starts from zero, reaches a maximum, and then exhibits a very slow linear decrease of $0.8 \times 10^{9} \mathrm{~cm}^{-2}$ per hour. By contrast, the loss on the upper segment in the linear region near the end of the decay curve amounts to about twice this value.

When the charging procedure of the two segments is exchanged the behavior changes accordingly. This demonstrates that the charging history influences the elec-

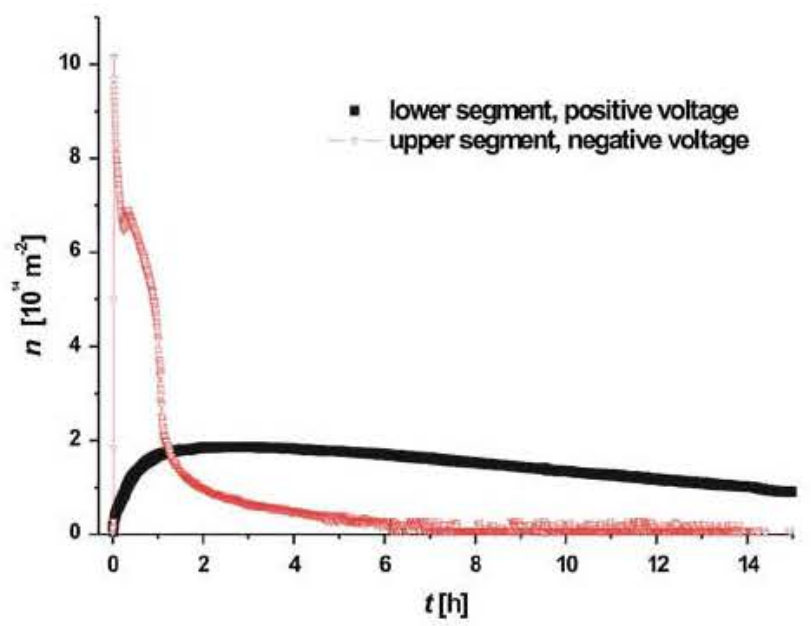

Fig. 2 Electron density as a function of time for two electrode segments with different charging history. Here, the upper segment was charged directly from a filament while the lower segment was charged by moving electrons from the upper to the lower segment (Color figure online) 
tron loss through the liquid film. One interpretation for this behavior could be electron transport of SSE through the core of quantized vortices in the film [15]. Charging the He film directly from a filament generates a perturbation which could give rise to the additional formation of quantized vortices. Charging a film sideways by pulling the thermalized electrons from the upper segment, on the other hand, should be much less perturbative. Since no temperature dependence of the electron loss was observed between 1.0 and $1.7 \mathrm{~K}$, a thermal mechanism for this process is unlikely. Whether it is of quantummechanical nature, e.g., tunneling of SSE through vortex cores, will be the subject of further investigations. However, our results demonstrate that smoother metallic substrates are required to reach high electron densities for significant periods of time.

\subsection{SSE on Films in Confined Geometry}

On micro- and nanostructured substrates, a mesoscopic meniscus forms, termed as a "suspended helium film" [16]. There exist a number of investigations of strong electron correlation and Wigner crystallization effects for suspended films in channels, including electron lane formation and the melting of the Wigner crystal [17-19]. In such microchannel geometries, the stability of the liquid surface is increased compared to the bulk surface not by van der Waals forces, but by surface tension.

Figure 3 shows the SSE current in a $10 \mu \mathrm{m}$-wide channel as a function of electron density $(T=0.95 \mathrm{~K})$. At low densities, the fluid region and the transition to the Wigner crystal, marked by a decrease in the SSE current, are clearly discernible. Upon further increase of the electron density, a sudden irreversible loss of the current signal points toward an ehd instability at $\sim 10^{10} \mathrm{~cm}^{-2}$. A similar limit has been observed in a

Fig. 3 Transport measurement of SSE confined in a $10 \mu \mathrm{m}$-wide channel. A back-gate voltage $V_{b g}$ is used to control the electron density on the channel. The density at which the SSE form a Wigner crystal is indicated by the arrow. SSE are lost irreversibly from the device for $n \sim 10^{10} \mathrm{~cm}^{-2}$

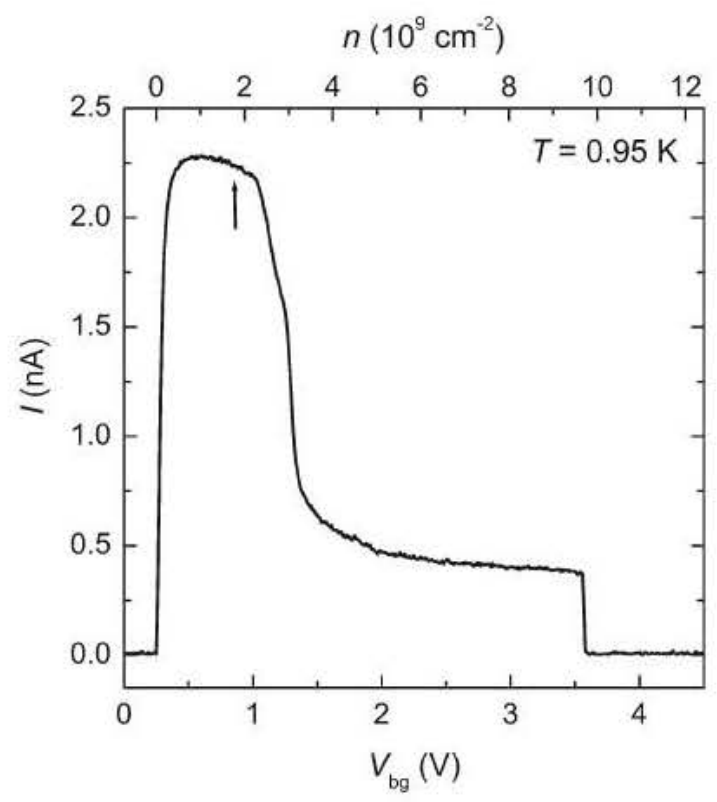


microchannel device by Shaban et al. [20]. We conclude that, while these microstructured substrates allow higher densities than achieved on bulk helium, nanoscale control of the suspended helium film is required in order to achieve densities approaching the quantum melting regime.

\section{Conclusions}

We have discussed several approaches for stabilizing the surface of liquid helium in order to reach much higher densities of SSE than on bulk He, which would allow one to study novel features of the SSE system such as the quantum melting transition. Experiments with helium films on insulating, metallic and microstructured surfaces show that it is possible to suppress the dimple instability on bulk helium and to go beyond the maximum density of $2 \times 10^{9} \mathrm{~cm}^{-2}$. However, up to now densities reaching $10^{11} \mathrm{~cm}^{-2}$ could be realized in only a few experiments. At such elevated densities new loss mechanisms come into play, which so far have not been investigated in detail, like the ehd instability in finite geometry and the transport of individual electrons through the film, possibly at weak spots like pinned quantized vortices. Although the high-density regime of SSE is thus still an experimental challenge, the preliminary results show that it should be possible to study quantum effects of electrons on $\mathrm{He}$ films more extensively.

Acknowledgments This work was partly supported by JSPS KAKENHI Grant No. 24000007, and by the Taiwan Ministry of Science and Technology (MOST) through the MOE ATU Program and Grant Nos. NSC 102- 2112-M-009-015 and MOST 103-2112-M-009-017. We thank A. Dahm for helpful discussion and J. Engelhardt for contributions to the experiments.

\section{References}

1. E. Andrei, Two-Dimensional Electron Systems on Helium and Other Cryogenic Substrates (Kluwer Academic, Dordrecht, 1997)

2. F.M. Peeters, P.M. Platzman, Phys. Rev. Lett. 50, 2021 (1983)

3. M. Wanner, P. Leiderer, Phys. Rev. Lett. 42, 315 (1979)

4. U. Albrecht, P. Leiderer, Europhys. Lett. 3, 705 (1987)

5. J. Tempere, I.F. Silvera, J.T. Devreese, Phys. Rev. B 67, 035402 (2003)

6. W. Guo, D. Jin, H.J. Maris, Phys. Rev. B 78, 014511 (2008)

7. E.M. Joseph, V. Vadakkumbatt, A. Pal, A. Ghosh, J. Low Temp. Phys. 175, 78 (2014)

8. K. Kajita, J. Phys. Soc. Jpn. 54, 4092 (1985)

9. H. Etz, W. Gombert, W. Idstein, P. Leiderer, Phys. Rev. Lett. 53, 2567 (1984)

10. H.-W. Jiang, M.A. Stan, A.J. Dahm, Surf. Sci. 196, 1-3 (1988)

11. G. Mistura, T. Günzler, S. Neser, P. Leiderer, Phys. Rev. B 56, 8360 (1997)

12. T. Günzler, B. Bitnar, G. Mistura, S. Neser, P. Leiderer, Surf. Sci. 361/362, 831 (1996)

13. A. Angrik, J. Faustein, P. Klier, J. Leiderer, Phys. Low Temp. Phys. 137, 335-344 (2004)

14. A. Faustein, Diploma thesis, University of Konstanz (2004)

15. F.M. Ellis, L. Li, Phys. Rev. Lett. 71, 1577 (1993)

16. A. Valkering, J. Klier, P. Leiderer, Phys. B 284, 172-173 (2000)

17. D.G. Rees, I. Kuroda, C.A. Marrache-Kikuchi, M. Höfer, P. Leiderer, K. Kono, Phys. Rev. Lett. 106, 026803 (2011)

18. H. Ikegami, H. Akimoto, K. Kono, Phys. Rev. Lett. 102, 046807 (2009)

19. H. Ikegami, H. Akimoto, D.G. Rees, K. Kono, Phys. Rev. Lett. 109, 236802 (2012)

20. F. Shaban, T. Lorentz, R. Rau, M. Ashari, D.G. Rees, K. Kono, E. Scheer, P. Leiderer, J. Phys. 568, 012008 (2014) 